\title{
The CDF MiniPlug Calorimeters at the Tevatron
}

\author{
K. Goulianos, M. Gallinaro, K. Hatakeyama, S.Lami*, C. Mesropian, K. Terashi \\ Department of Experimental Physics, The Rockefeller University, New York, New York 10021, USA
}

Two MiniPlug calorimeters, designed to measure the energy and lateral position of particles in the pseudorapidity region of $3.6<|\eta|<5.1$ of the CDF detector, have been installed as part of the Run II CDF upgrade at the Tevatron collider. Detector performance and first results from $\bar{p} p$ collision data are presented.

\section{INTRODUCTION}

The CDF diffractive physics program relies on two MiniPlug (MP) calorimeters, which can detect both charged and neutral particles, to measure the event energy flow in the very forward rapidity region on opposite sides of the interaction point. The performance of a MP prototype is published in 1, while the final detector assembly and the results from a cosmic ray test are reported in 2].

\section{DETECTOR}

The MPs consist of alternating layers of lead plates and liquid scintillator read out by WaveLength Shifting (WLS) fibers (Fig. 1). The fibers are perpendicular to the lead plates and parallel to the proton/antiproton beams, and are read out by Multi-Channel PhotoMultiplier Tubes (MCPMTs). The 16-channel Hamamatsu R5900 MCPMTs have a quartz window which improves the radiation hardness. Each MP is housed in a cylindrical steel vessel $26^{\prime \prime}$ in diameter and has a $5^{\prime \prime}$ hole concentric with the vessel to accommodate the beam pipe. The depth of a MP is 32 radiation lengths and 1.3 interaction lengths. The short hadronic depth limits the lateral spread of the showers. The MPs have a novel towerless geometry with no dead regions, due to the lack of internal boundaries, and towers are formed by combining the desired number of fibers. The centroid of the tower pulse height provides the posi-

${ }^{*}$ Corresponding author. Tel.: +1-212-327-8832; fax: +1212-327-7786. E-mail address: lami@fnal.gov (S. Lami) tion of the shower initiating particle.

The tower design is based on an hexagon geometry. Holes in the lead plate are conceptually grouped in hexagons, each with six holes. The six fibers inserted in the holes of a hexagon are grouped together and are viewed by one MCPMT channel. A seventh fiber, which is clear and car-

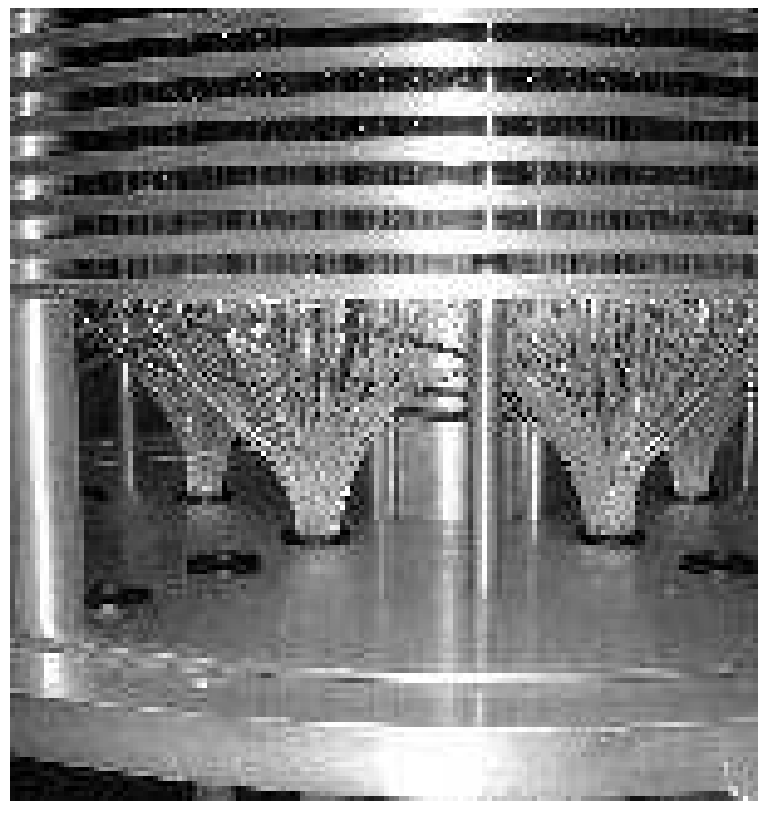

Figure 1. Fiber routing in the MiniPlug.

ries the light from a calibration LED, is also read out by each MCPMT pixel to allow a periodical monitoring of the MCPMT response. The MCPMT outputs are added to form 84 calorimeter towers per MP, organized in four concentric 
rings around the beam pipe. The entire MCPMT can also be read out through the last dynode output to provide trigger information. Each MP has a total of 18 trigger towers, arranged in three rings.

\section{CALIBRATION AND RUN II DATA}

Cosmic ray muons were used to test one $60^{\circ}$ wedge of the east MP. The cosmic ray trigger fired on a 2-fold coincidence of scintillation counter paddles located on top and at the bottom of the MP vessel, placed with towers pointing upward. The single photoelectron response was measured using a randomly gated signal from a ${ }^{60} \mathrm{Co}$ source. The response to a minimum ionizing particle was found to be above 100 photoelectrons, exceeding the design specifications.

Although a precise energy calibration is not crucial to the analysis of diffractive processes, an attempt was made to estimate the energy of jets and particles using colliding beam data from Run II. For each tower, the ADC count distribution of a sample of minimum bias events can be fitted well by a falling exponential curve (Fig. 2] top). A Monte Carlo simulation was then used to calibrate the pseudorapidity dependence of the tower-by-tower response. Due to pile-up effects at larger rapidity regions, a luminosity dependence of the ADC count distribution slope is observed (Fig. 2 bottom). A linear fit describes well the data, with the slope decreasing with increasing values of instantaneous luminosity. The particle multiplicity is measured by counting clusters of towers with energy above noise (Fig. 33).

The MPs are presently an integral part of the CDF detector, collecting good quality data for further exploring the realm of diffractive physics.

\section{REFERENCES}

1. K. Goulianos and S. Lami, Nucl. Instrum. Methods A430 (1999) 34.

2. K. Goulianos et al., Nucl. Instrum. Methods A496 (2003) 333.

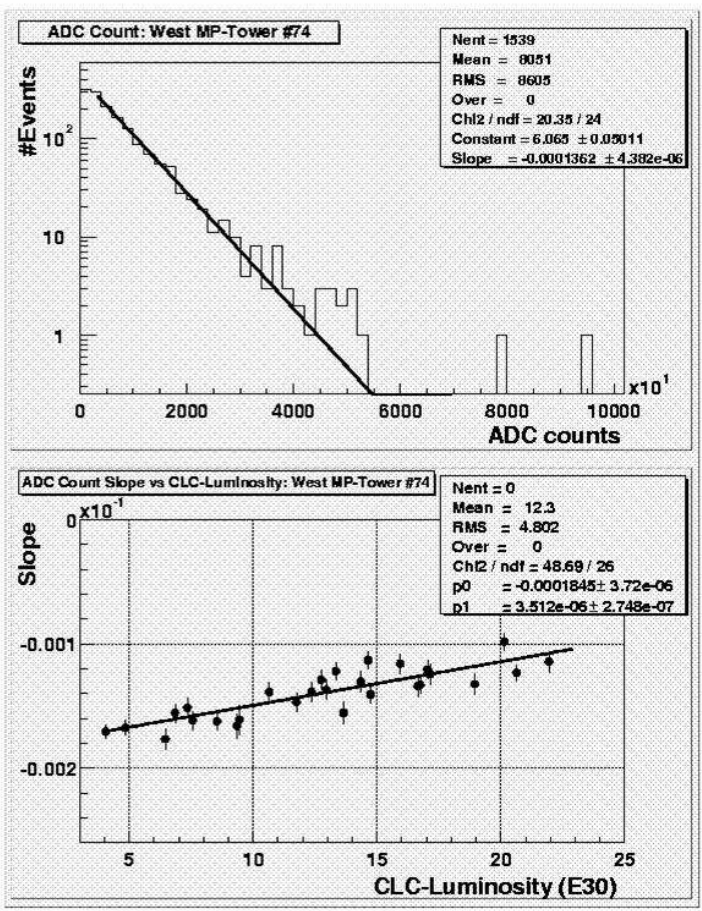

Figure 2. The ADC count distribution of the data can be fitted well by a falling exponential curve $(t o p)$. The absolute slope values decrease with increasing luminosity due to event pile-up (bottom). Data are shown for Tower 74 of the west MP.

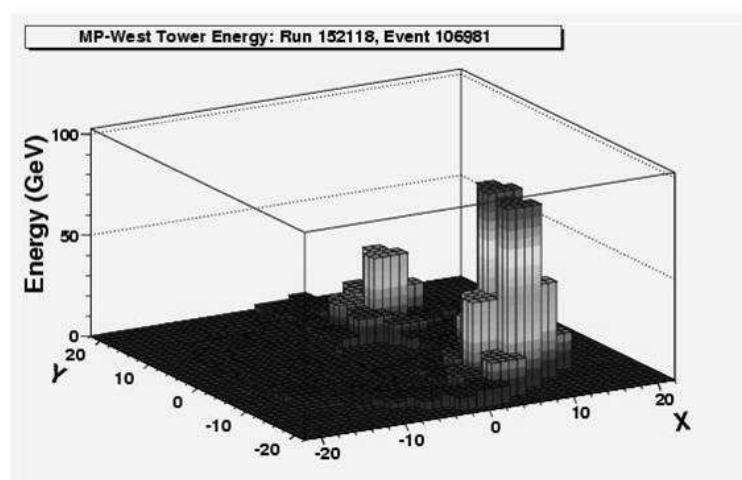

Figure 3. Two hadron event in the west MP. 